\title{
Formal Conditions on Quantum Teleportation
}

\author{
Agus Purwanto, Heru Sukamto, and Lila Yuwana* \\ Department of Physics, Institut Teknologi Sepuluh Nopember, Surabaya, Jawa Timur 60111, \\ Indonesia; purwanto@physics.its.ac.id, herusukamto@physics.its.ac.id, lila@physics.its.ac.id
}

\begin{abstract}
Objectives: To formulate the most elementary requirement to realise quantum teleportation. Methods: By expanding procedure to define the capability of general two qubit quantum states to realise quantum teleportation. Findings: By structuring the general form of two qubit and measurement states, we can exhibit the capabilty both separable two qubit states and entangled two qubit states to realise quantum teleportation. Next, we also show the measurement procedure and the unitary operator required to reconstruct a single qubit delivered into correct state. Application/Improvement: The methods exhibited in this paper have relatively simple procedure and very beneficial to improve our last procedure proposed beforehand.
\end{abstract}

\section{Keywords: Entangled States, Separable States, Quantum Channels, Quantum Teleportation}

\section{Introduction}

The research of quantum teleportation theoretically coined by ${ }^{1}$ lead to sustained reseach theoretically and experimentally. In their paper, Bennet utilisedone of Einstein-Podolsky-Rosen (EPR) statesas a quantum channel. In experimental aspect, several essential experiments of quantum teleportation have been conducted both in laboratory and in free space. Firstly have successfully developed quantum teleportation using polarised photons as a quantum channel produced by applying parametric down conversion type II in a laboratory ${ }^{1} \mathrm{In}^{3}$ performed a quantum teleportation for about $2.5 \mathrm{~m}$ in a laboratoryby utilising linear and elliptic polarisation of photons. Thirdly, Rupert ${ }^{4}$. designed a quantum teleportation that polarised photons were delivered along $800 \mathrm{~m}$ of fibre optics in $^{5-6}$. Teleported polarised photons in free spaceguided by telescopes in the distance of $16 \mathrm{~km}$ and $143 \mathrm{~km}$ in 2010 and 2012, respectively. On the other side, there is a sustained research theoreticallyon quantum teleportation. One of intriguing topics on quantum teleportation is the capability of quantum channel to realise teleportation. Have successfully structured quantum teleportation system by introducing the requirements of a teleportation. Furthermoreindicated a quantum channel whether is able to realise teleportation or not by examiningtransformation operator determinant. If the determinant of operator is zero, then quantum teleportation cannot be realised ${ }^{7}$. Revealed the relation between the transformation operator determinant and Stochastic Local Operation and Classical Communication (SLOCC) invariant are also able to be used to identify the quantum channels capability to realise quantum teleportation ${ }^{8}$. In $^{9}$ examinedquantum teleportation using Werner-like state as a resource ${ }^{9}$. In $^{10}$ proposedentanglement identification by using Schmidt decomposition and also identification of the capability of channel states to realise quantum teleportation ${ }^{10}$.

We intend to expand quantum teleportation proposed by $^{1}$, which was only utilising maximally entangled states as a quantum channels. Henceforth, this article examine capabilities of general separable and entangled states to realise quantum teleportation. This article also proposes to improve research ${ }^{10}$, especially in the identification of capabilities of quantum channelsto realise quantum teleportation. This is because reported thatthere are entangled states that are not able to realise quantum teleportation ${ }^{10}$. We have revealed that all types of entangled states are able

${ }^{*}$ Author for correspondence 
to realise quantum teleportation. Furthermore, we exhibit elementary requirements to realise quantum teleportation by applying general entangled states as quantum channels whereas Bennet utilised only maximally entangled states, i.e. EPR states.

The following sections of this article are arranged as follows. In Section 2, we show how general separable statesfail to realise quantum teleportation. Next, the viability of general entangled states to realise quantum teleportation are proved successfullyin Section 3. Finally, the conclusions of capability of both general separable and entangled states to realise are stated obviously in Section 4.

\section{General Separable States Collap to Realise Quantum Teleportation}

Suppose Alice sendsan unknown informationto Bob in the form of single-qubit state (abbreviated as particle 1$)^{1}$

$$
|\chi\rangle_{a}=x_{0}|0\rangle_{a}+x_{1}|1\rangle_{a}
$$

Where $\left|x_{0}\right|^{2}+\left|x_{1}\right|^{2}=1$. If an information $|\chi\rangle_{a}$ is sent to Bob by using separable state as a quantum channel then:

$$
\begin{aligned}
|\varphi\rangle_{A B}= & \left(a_{0}|0\rangle+a_{1}|1\rangle\right)_{A} \otimes\left(b_{0}|0\rangle+b_{1}|1\rangle\right)_{B} \\
= & \left(a_{0} b_{0}|00\rangle+a_{0} b_{1}|01\rangle\right. \\
& \left.+a_{1} b_{0}|10\rangle+a_{1} b_{1}|11\rangle\right)_{A B}
\end{aligned}
$$

Wheresubscript $a, A$, and $B$ in Eq. (1) and (3) denote the owner of particle. It means particle a in $|\chi\rangle$ belongs to Alice and particle $A$ and $B$ in $|\varphi\rangle$ are possessed by Alice and Bob. According to the procedure of quantum teleportation, by combining information sent by Alice in Eq. (1) and the quantum channel in Eq. (2), we have:

$$
\begin{gathered}
|\psi\rangle_{a A B}=|\chi\rangle_{a} \otimes|\varphi\rangle_{A B} \\
=x_{0}\left(a_{0} b_{0}|000\rangle+a_{0} b_{1}|001\rangle\right. \\
\left.+a_{1} b_{0}|010\rangle+a_{1} b_{1}|011\rangle\right) \\
+x_{1}\left(a_{0} b_{0}|100\rangle+a_{0} b_{1}|101\rangle\right. \\
\left.+a_{1} b_{0}|110\rangle+a_{1} b_{1}|111\rangle\right)
\end{gathered}
$$

By combining Alice's state and the quantum channel lead to the annihilation of the origin state sent by Alice. In other words, the quantum teleportation is suitable with the requirement of non-cloning theorem ${ }^{11,12}$.

Furthermore, Alice utilise a measurement on the compound state by applying another quantum state

$$
|\pi\rangle=m_{0}|00\rangle+m_{1}|01\rangle+m_{2}|10\rangle+m_{3}|11\rangle
$$

Where, $\left|m_{0}\right|^{2}+\left|m_{1}\right|^{2}\left|m_{2}\right|^{2}+\left|m_{3}\right|^{2}=1$. The information received by Bob can be obtained by a measurement process of:

$$
\begin{gathered}
\langle\pi|\otimes I| \Psi\rangle=\left|\chi^{\prime}\right\rangle_{B}=\left[\left(m_{0} x_{0}+m_{2} x_{1}\right) a_{0} b_{0}\right. \\
\left.+\left(m_{1} x_{0}+m_{3} x_{1}\right) a_{1} b_{0}\right]|0\rangle \\
+\left[\left(m_{0} x_{0}+m_{2} x_{1}\right) a_{0} b_{1}+\left(m_{1} x_{0}+m_{3} x_{1}\right) a_{1} b_{1}\right]|1\rangle
\end{gathered}
$$

A realisation of quantum teleportation can be attained if Eq. (5) is able to be expressed into Eq. (1), hence let $m_{2}=$ $m_{3}=0$, then we obtain:

$$
\begin{aligned}
& \left|\chi^{\prime}\right\rangle_{B}=\left(m_{0} a_{0} b_{0}+m_{1} a_{1} b_{0}\right) x_{0}|0\rangle \\
& \quad+\left(m_{0} a_{0} b_{1}+m_{1} a_{1} b_{1}\right) x_{0}|1\rangle
\end{aligned}
$$

Obviously, Bob are not able to receive the precise state that is the same as the origin state in Eq. (1) because there is no amplitude $\mathrm{x}_{1}$ remains in Eq. (6). Correspondingly, if we choose $m_{0}=m_{1}=0$, then the coefficients of and are $x_{1}$ without $\mathrm{x}_{0}$. It means that the separable states cannot realise quantum teleportation. In the following paragraph, we deliver clear example to show why a separable states are not able to realise quantum teleportation mathematically.

\section{Example 1}

Let a quantum channel as follows:

$$
|\varphi\rangle_{A B}=\frac{1}{2}(|00\rangle+|01\rangle+|10\rangle+|11\rangle)
$$

then,

$$
\begin{gathered}
|\psi\rangle_{a A B}=\frac{x_{0}}{2}(|000\rangle+|001\rangle+|010\rangle+|011\rangle) \\
+\frac{x_{1}}{2}(|100\rangle+|101\rangle+|110\rangle+|111\rangle)
\end{gathered}
$$


And

$$
\begin{aligned}
& \left|\chi^{\prime}\right\rangle_{B} \\
& \quad=\frac{1}{2}\left\{\left(m_{0} x_{0}+m_{2} x_{1}\right)+\left(m_{1} x_{0}+m_{3} x_{1}\right)\right\}(|0\rangle+|1\rangle)
\end{aligned}
$$

Obviously, there is no value of to convert $\left|\chi^{\prime}\right\rangle_{B}$ received by Bob into the expression that is the same as original quantum state sent by Alice $\left(|\chi\rangle_{a}\right)$.

\section{Example 2}

Let a quantum channel as follows:

$$
|\varphi\rangle_{A B}=\frac{1}{2}(0.8|00\rangle+0.6|01\rangle)
$$

then,

$$
\begin{aligned}
& |\psi\rangle_{a A B}=x_{0}(0.8|000\rangle+0.6|001\rangle) \\
& \quad+x_{1}(0.8|100\rangle+0.6|101\rangle)
\end{aligned}
$$

And

$$
\left|\chi^{\prime}\right\rangle_{B}=\left(m_{0} x_{0}+m_{2} x_{1}\right)(0.8|0\rangle+0.6|1\rangle)
$$

Obviously, there is no value of to convert $\left|\chi^{\prime}\right\rangle_{B}$ received by Bob into the expression that is the same as original quantum state sent by Alice $\left(|\chi\rangle_{a}\right)$.

\section{General Entangled State Succeeds to Realise Quantum Teleportation}

Now, we attempt to vindicatethat general entangled states as a quantum channelsare able to realise quantum teleportation. Next, if Alice deliversa single-qubit in Eq. (1) by using general form of two-qubit quantum channel

$$
|\varphi\rangle_{A B}=\left(c_{0}|00\rangle+c_{1}|01\rangle+c_{2}|10\rangle+c_{3}|11\rangle\right)_{A B}
$$

Where $\left|c_{0}\right|^{2}+\left|c_{1}\right|^{2}+\left|c_{2}\right|^{2}+\left|c_{3}\right|^{2}=1$ and each coefficient/amplitudo cannot be separated into tensor product as well as Eq. (2), and we obtain the general form of compound system state as follows

$$
\begin{gathered}
|\psi\rangle_{a A B}=|\chi\rangle_{a} \otimes|\varphi\rangle_{A B} \\
=x_{0}\left(c_{0}|000\rangle+c_{1}|001\rangle+c_{2}|010\rangle+c_{3}|011\rangle\right) \\
+x_{1}\left(c_{0}|100\rangle+c_{1}|101\rangle+c_{2}|110\rangle+c_{3}|111\rangle\right)
\end{gathered}
$$

Furthermore, Alice utilisesa measurement Eq. (4) on the compound state by applying another quantum state by operating:

$$
\begin{aligned}
& \langle\pi|\otimes I| \Psi\rangle=\left|\chi^{\prime}\right\rangle_{B} \\
& \quad=\left[\left(m_{0} c_{0}+m_{1} c_{2}\right) x_{0}+\left(m_{2} c_{0}+m_{3} c_{2}\right) x_{1}\right]|0\rangle \\
& +\left[\left(m_{0} c_{1}+m_{1} c_{3}\right) x_{0}+\left(m_{2} c_{1}+m_{3} c_{3}\right) x_{1}\right]|1\rangle
\end{aligned}
$$

A realisation of quantum teleportation can be attained if Eq. (9) is able to be expressed into Eq. (1), therefore we select:

$$
m_{0}=-\frac{c_{2}}{c_{1}} m_{1} ; m_{2}=-\frac{c_{2}}{c_{0}} m_{3}
$$

then we obtain:

$$
\begin{gathered}
\left|\chi^{\prime}\right\rangle_{B}=\left(-\frac{c_{3}}{c_{1}} c_{0}+c_{2}\right) m_{1} x_{0}|0\rangle \\
+\left(-\frac{c_{2}}{c_{0}} c_{1}+c_{3}\right) m_{3} x_{1}|1\rangle
\end{gathered}
$$

In order to suit into Eq. (1) then we choose:

$$
m_{1}=\frac{1}{\left(-\frac{c_{3}}{c_{1}} c_{0}+c_{2}\right)} \text { and } m_{1}=\frac{1}{\left(-\frac{c_{2}}{c_{0}} c_{1}+c_{3}\right)}
$$

Eventually, Eq. (11) can be expressed into Eq. (1) as follows:

$$
\left|\chi^{\prime}\right\rangle_{B}=x_{0}|0\rangle+x_{1}|1\rangle
$$


Therefore, Bob are able to reconstruct the information that is exactly the same as Eq. (1) by operating

$$
\sigma_{B}\left|\chi^{\prime}\right\rangle_{B}=I\left|\chi^{\prime}\right\rangle_{B}=x_{0}|0\rangle+x_{1}|1\rangle
$$

An other option is $m_{0}=-\frac{c_{2}}{c_{0}} m 1 ; m_{2}=-\frac{c_{3}}{c_{1}} m_{3}$, then

$$
\begin{gathered}
\left|\chi^{\prime}\right\rangle_{B}=\left(c_{2}-\frac{c_{3}}{c_{1}} c_{0}\right) m_{3} x_{1}|0\rangle \\
+\left(c_{3}-\frac{c_{2}}{c_{0}} c_{1}\right) m_{1} x_{0}|1\rangle
\end{gathered}
$$

and

$$
\left|\chi^{\prime}\right\rangle_{B}=\sigma_{B}\left(x_{0}|0\rangle+x_{1}|1\rangle\right)
$$

Eventually, Bob is able to reconstruct the information that is exactly the same as Eq. (1) by operating:

$$
\sigma_{B}\left|\chi^{\prime}\right\rangle_{B}=\sigma_{x}\left|\chi^{\prime}\right\rangle_{B}
$$

It means the plausible conclusion stated by Yuwana et. al has to be improved into following explanation. According to Eq. (7-14), we reveal that the general entangled state is able to realise quantum teleportation. This is because a state received by Bob always can be reconstructed into initial state sent by Alice. In the next paragraph, we deliver clear example to show why a separable state are not able to realise quantum teleportation mathematically.

\section{Example 3}

Let we apply quantum channel as follows:

$$
\begin{aligned}
& |\varphi\rangle_{A B}=\frac{1}{2}(|00\rangle+|01\rangle+|10\rangle-|11\rangle) \\
& \text { then we have } c_{0}=c_{1}=c_{2}=-c_{3}=\frac{1}{2}
\end{aligned}
$$

According to Eq. (11), we obtain:

$$
\begin{aligned}
& \left|\chi^{\prime}\right\rangle_{B}=\frac{1}{2}\left[\left(m_{0} x_{0}+m_{2} x_{1}\right)(|0\rangle+|1\rangle)\right. \\
& \left.\quad+\left(m_{1} x_{0}+m_{3} x_{1}\right)(|0\rangle+|1\rangle)\right]
\end{aligned}
$$

If Alice performs measurement of $m_{0}=m_{1}=m_{2}$ $=-m_{3}=\frac{1}{2}$, then Bob receives:

$$
\left|\chi^{\prime}\right\rangle_{B}=\frac{1}{2}\left(x_{0}|0\rangle+x_{1}|1\rangle\right)=\sigma_{B}\left(x_{0}|0\rangle+x_{1}|1\rangle\right)
$$

Table 1. Measurements values of example 1 and related unitary operator to realise quantum teleportation

\begin{tabular}{|l|l|l|l|l|}
\hline No. & Quantum Channel & $m_{0}, m_{1}, m_{2}, m_{3}$ & $\left|\chi^{\prime}\right\rangle_{B}$ & $\sigma_{B}$ \\
\hline \multirow{2}{*}{1} & $|\varphi\rangle_{A B}=\frac{1}{2}(|00\rangle+|01\rangle+|10\rangle-|11\rangle)$ & $m_{0}=-m_{1}=m_{2}=m_{3}=\frac{1}{2}$ & $\frac{1}{2}\left(x_{0}|0\rangle-x_{1}|1\rangle\right.$ & $2 \sigma_{z}$ \\
\cline { 3 - 5 } & $m_{0}=m_{1}=m_{2}=-m_{3}=\frac{1}{2}$ & $\frac{1}{2}\left(x_{0}|0\rangle+x_{1}|1\rangle\right.$ & $2 I$ \\
\cline { 3 - 5 } & $m_{0}=-m_{1}=m_{2}=m_{3}=\frac{1}{2}$ & $\frac{1}{2}\left(x_{0}|0\rangle-x_{1}|1\rangle\right.$ & $2 \sigma_{z}$ \\
\cline { 3 - 5 } & $m_{0}=-m_{1}=m_{2}=m_{3}=\frac{1}{2}$ & $\frac{1}{2}\left(x_{0}|0\rangle-x_{1}|1\rangle\right.$ & $2 \sigma_{z}$ \\
\hline
\end{tabular}


Table 2. Measurements values of example 2 and related unitary operator to realise quantum teleportation

\begin{tabular}{|c|c|c|c|c|c|c|c|}
\hline \multirow{2}{*}{$\begin{array}{l}\text { No. } \\
1 .\end{array}$} & \multirow{2}{*}{$\begin{array}{l}\text { Quantum Channel } \\
\begin{array}{c}|\varphi\rangle_{A B}=0.5|00\rangle+0.5|01\rangle \\
+0.7|10\rangle+0.1|11\rangle\end{array}\end{array}$} & \multicolumn{4}{|c|}{$m_{0}, m_{1}, m_{2}, m_{3}$} & \multirow{2}{*}{$\frac{\left|\chi^{\prime}\right\rangle_{B}}{0.3\left(-x_{0}|0\rangle+x_{1}|1\rangle\right.}$} & \multirow{2}{*}{$\begin{array}{l}\sigma_{B} \\
-\frac{10}{3} \sigma_{z}\end{array}$} \\
\hline & & 0.1 & -0.5 & 0.7 & -0.5 & & \\
\hline & & 0.1 & -0.5 & -0.7 & 0.5 & $-0.3\left(-x_{0}|0\rangle+x_{1}|1\rangle\right.$ & $-\frac{10}{3} I$ \\
\hline & & -0.1 & 0.5 & 0.7 & -0.5 & $0.3\left(x_{0}|0\rangle+x_{1}|1\rangle\right.$ & $\frac{10}{3} I$ \\
\hline & & -0.1 & 0.5 & -0.7 & 0.5 & $0.3\left(x_{0}|0\rangle-x_{1}|1\rangle\right.$ & $\frac{10}{3} \sigma_{z}$ \\
\hline
\end{tabular}

Eventually, quantum teleportation can be realised if Bob apllies unitary operator $\sigma_{B}=2 I$. Finally, various values of $m_{0}, m_{1}, m_{2}$, and $m_{3}$ that can be applied to realise quantum teleportation are listed in the (Table 1).The different selections of $m_{0}, m_{1}, m_{2}$, and $m_{3}$ lead to various unitary operators to reconstruct $\left|\chi^{\prime}\right\rangle_{B}$ into $|\chi\rangle_{a}$.

\section{Example 4}

Let we apply quantum channel as follows:

$$
|\varphi\rangle_{A B}=0.5|00\rangle+0.5|01\rangle+0.7|10\rangle+0.1|11\rangle
$$

then we have $C_{0}=C_{1}=0.5 ; C_{2}=0.7 ; C_{3}=0.1$

According to Eq. (9), we obtain:

$$
\begin{gathered}
\left|\chi^{\prime}\right\rangle_{B}=\left\{\left(0.5 m_{0}+0.7 m_{1}\right) x_{0}+\left(0.5 m_{2}+0.7 m_{3}\right)\right\}|0\rangle \\
\quad+\left\{\left(0.5 m_{0}+0.1 m_{1}\right) x_{0}+\left(0.5 m_{2}+0.1 m_{3}\right)\right\}|1\rangle
\end{gathered}
$$

If Alice performs measurement of $m_{0}=0.1$, $m_{1}=-0.5, m_{2}=0.7, m_{3}=-0.5$, then Bob receives:

$$
\left|\chi^{\prime}\right\rangle_{B}=\left(0.3\left(-x_{0}|0\rangle+x_{1}|1\rangle\right)\right)=\sigma_{B}\left(x_{0}|0\rangle+x_{1}|1\rangle\right)
$$

Eventually, quantum teleportation can be realised if Bob apllies unitary operator $\sigma_{B}=-\frac{10}{3} \sigma_{z}$. Finally, various values of $m_{0}, m_{1}, m_{2}$, and $m_{3}$ that can be applied to realise quantum teleportation are listed in the (Table 2). The different selections of $m_{0}, m_{1}, m_{2}$, and $m_{3}$ lead to various unitary operators to reconstruct $\left|\chi^{\prime}\right\rangle_{B}$ into $|\chi\rangle_{a}$.

\section{Conclusion}

The methods to identify capabilities of general separable state and general entangled state to realise teleportation is revealed. We show that all separable states are not able to realisequantum teleportation whereas all entangled states are able to ensure the viability of quantum teleportation. This results also improve the conclusion proposed by Yuwana et. al., i.e. there were entangled states could not realise quantum teleportation. Finally, we also deliver examples to explain more explicit calculations.

\section{Acknowledgement}

The authors acknowledge to The Ministry of Research, Technology, and Higher Education of The Republic of Indonesia for reseacrh funding of Hibah Penelitian Doktor Baru (Research Grant Program for New Doctors) 2018 according to Contract No. 1199/PKS/ITS/2018.

\section{References}

1. Bennet CH, Brassard G, Crepeau C, Jozza R, Peres A, Wootters WK. Teleporting an unknown quantum state via dual classical and Einstein-Podolsky-Rosen Channels, Physical Review Letters. 1993; 70(13):1895-99. crossref. PMid: 10053414.

2. Bouwmeester D, Pan JW, Mattle K, Eibi M, Weinfurter H, Zeilinger A. Experimental quantum teleportation, Nature. 1997; 390:575-79. crossref. 
3. Boschi D, Branca S, Martini FD, Hardy L,Propescu S. Experimental realization of teleporting an unknown pure quantum state via dual classical and EinsteinPodolsky-Rosen channels, Physical Review Letters. 1998; 80(6):1121-25. crossref.

4. Ursin R, Jennewein $\mathrm{T}$, Aspelmeyer M, Kaltenbaek R, Lindenthal M. Communications:Quantum teleportation across the Danube, Nature. 2004; 430:849. crossref. PMid:15318210.

5. Jin XM, Ren J-G, Yang B, Yi Z-H, Zhou F. Experimental free-space quantum teleportation, Nature Photonics. 2010; 4:376-81. crossref.

6. Ma X-S, Herbst T, Scheidl T, Wang D, Kropatschek S. Quantum teleportation over 143kilometres using active feed-forward, Nature. 2012; 489:269-73. crossref. PMid:22951967.

7. Zha X-W, Huang C-B. The expansion of orthogonal complete set and teleportation of an arbitary two-qubit state, Acta Physica Sinaca. 2007; 56(4):1875-80.
8. Zha X-W, Ren KF. General relation between the transformation operator and an invariant under stochastic local operations and classical communication in quantum teleportation, Physical Review A. 2008; 77:014306. crossref.

9. Verma V,Prakash H. Quantum teleportation of single qubit mixed information state with Werner-like state as resource. In: 12th International Conference on Fiber Optics and Photonics. Kharagpur; 2014. 1-12 crossref.

10. Yuwana L, Purwanto A, Endarko,Januriyanto D. Entanglement identification of arbitrary two-qubit quantum channels and the capabilities to realise quantum teleportation, Indian Journal of Science and Technology. 2017; 10(43):1-4. crossref.

11. Nakahara M,Ohmi T. Quantum computing, from linear algebra to physical realization. CRC Press: Taylor and Francis Group; 2008. p. 440. crossref.

12. Nielsen MA, Chuang IL. Quantum Computation and Quantum Information. Massachusetts Institute of Technology. New York: Cambridge University Press; 2010. crossref. 